\title{
Status of unchosen objects in discrimination learning by monkeys
}

\author{
DOUGLAS L. MEDIN \\ The Rockefeller University, New York, New York 10021
}

\begin{abstract}
Monkeys were given a mixture of discrimination training and choice trials. The choice involved a previously unchosen object selected from a trial where the chosen object had been rewarded vs. a previously unchosen object selected from a trial where the chosen object had not been rewarded. On these nondifferentially rewarded choice trials, monkeys exhibited a small but consistent preference for the previously unchosen object that had been associated with a rewarded outcome. The results showed that the outcome of a discrimination trial is associated to varying degrees with both the chosen and unchosen objects, but the mechanism by which unchosen objects are associated with trial outcomes remains uncertain.
\end{abstract}

Primates are notorious for their improvement in the rate of solving discrimination learning problems as a function of practice. Over a series of a few hundred discrimination problems monkeys move from slow "trial and error" learning to "insightful" learning, where most discriminations are solved after a single trial (Harlow, 1949). Therefore, common sense dictates that, in the course of learning set training, monkeys learn the structure of the discrimination task, including the fact that one of the two objects will be correct and rewarded and the other will be incorrect and nonrewarded.

If monkeys acquire the structure of discrimination, one might expect that they could infer the reward status of the unchosen stimulus object. At least three experiments (Behar, 1962; Fitzgerald \& Davis, 1960; Leary, 1956) have given monkeys a series of problems where every few trials either the correct or incorrect stimulus was replaced by a new correct or incorrect stimulus. On these shifts, monkeys preferred the old incorrect object to a new object if they had not chosen the incorrect object in the previous set of trials. This occurred even though this strategy was never rewarded. The fact that the incorrect object had not been chosen previously indicates that it was not highly preferred, so something was learned about it during these preshift trials. If monkeys had inferred that the unchosen object was unrewarded (because the other, chosen object was being rewarded), they should have avoided the old incorrect stimulus objects on shift trials. On the other hand, any tendency to act on inferences may have been overshadowed by an even stronger tendency to approach familiar, or to avoid novel, stimulus objects.

An alternative explanation of the above results is that the outcome of a trial is associated in varying degrees with both the chosen and the unchosen objects.

This research was supported by United States Public Health Service Grants MH 25134 and MH 16100.
Unchosen incorrect objects may gain in attractiveness because of their (indirect) association with reward. Indeed, this assumption was made by Medin (1975) in his proposed model for the role of context in discrimination learning. To test this idea, the present experiment employed a paradigm similar to that used by Behar (1962), Fitzgerald and Davis (1960), and Leary (1956) except that the critical tests consisted of stimuli equated for familiarity.

\section{METHOD}

Subjects

The subjects were five 5- to 6-year-old jungle-born pigtailed monkeys (Macaca nemestrina) and two 3- to 4-year-old jungleborn rhesus monkeys (Macaca mulatta). All animals were learning set experienced and had 2-3 years experience on various types of discrimination problems. Immediately prior to the present experiment, the monkeys had been trained on delayed matching-to-sample problems, where the correct object on a choice test was always the object that had been rewarded most recently.

\section{Apparatus}

A Wisconsin General Test Apparatus (WGTA) was employed for testing. The two foodwells of the gray form board were spaced $15 \mathrm{~cm}$ apart, edge to edge. The 312 stimulus objects used in the experiment were selected from a pool of commonuse and manufactured objects.

\section{Procedure}

The basic test sequences involved three choice tests followed by three trials of regular discrimination training. For one of the first two choice trials, a raisin was placed under each object so that regardless of the monkey's choice, a reward would be forthcoming. For the other of the two choice trials, no reward was available. The rewarded trial was randomly determined but was balanced each day. Each of these initial two trials used new objects and the choices made determined which objects would be used on the third trial and which on the subsequent three discrimination learning trials. The chosen objects became the stimuli for the discrimination learning trials; the chosen object that had been rewarded became the correct object and the chosen object that had not been rewarded became the incorrect object. The position of the rewarded object for the three- 
Table 1

A Possible Sequence of Trials in the Experiment

\begin{tabular}{ccc}
\hline \multicolumn{3}{c}{ Choice Trials } \\
1 & A & B \\
- & C & + \\
2 & - & D \\
- & B & C \\
3 & - & - \\
- & Discrimination Trials & \\
4 & D & A \\
- & - & + \\
5 & D & A \\
- & - & + \\
6 & A & D \\
- & + & - \\
\hline
\end{tabular}

Note-The circles indicate the presumed choices on the first two trials. The initial choices determine which objects will be used on the other trials.

trial problems was randomly determined. The unchosen stimuli from the first two choice trials were paired on the third and critical choice trial. A random but balanced (within a day) $50 \%$ of these choice trials were rewarded. For all trials only a single response was allowed and the intertrial interval was approximately $12 \mathrm{sec}$. A hypothetical trial sequence is shown in Table 1. The circles indicate the presumed choices on the initial two trials.

Every six trials a new six-trial sequence using four new objects was begun. Six such sequences were given each day, for a total of 36 trials and 24 different stimulus objects. In all, 13 days of testing were given.

\section{RESULTS}

The main variable of interest concerns responses on the third choice trials of a sequence which pitted an unchosen object from a trial where the chosen object had been rewarded against an unchosen object from a trial where the chosen object had not been rewarded. Each of the stimuli had been presented once and had not been chosen, so there was no reason to expect differential choices based either on familiarity or stimulus preferences. Overall, $56.1 \%$ of the choice responses were to the object that had come from a rewarded trial. Six of the seven monkeys showed this preference and one monkey displayed no preference. Statistical tests confirm these trends. Because of a tester error, the critical choice data from the first day were not recorded and the analyses are based on the other 12 days of testing (broken into two 6-day blocks). An ANOVA indicated that the effect of prior reward and nonreward pairing was significant $[F(1,6)=6.1, p<.05]$ and that the interaction of Blocks of Testing by Prior Reward and Nonreward Pairs was not significant $(F<1)$. Early in the experiment we thought we noticed a tendency for the monkeys to choose the stimulus that had been presented most recently, but statistical analyses failed to confirm this impression. On the first differentially rewarded trial of discrimination training, which was Trial 4 in a sequence, monkeys averaged $79.1 \%$ correct responses, indicating that they had acquired information from the initial two trials of a sequence.

\section{DISCUSSION}

On a nondifferentially rewarded choice test, monkeys preferred a previously unchosen object that previously had been paired with a chosen rewarded object to a previously unchosen object that previously had been paired with a chosen nonrewarded object. This trend, which was small but consistent, is contrary to what might be expected if monkeys inferred that an unchosen object has the opposite valence from that of the chosen object. The results are consistent with the context theory, which assumes that the outcome of a trial is associated with both the chosen and the unchosen objects.

Other studies indicating that unchosen negative objects increase in attractiveness paired unchosen negative objects with new stimuli and thus did not equate the stimuli for familiarity. The fact that the effect observed in the present study was about half the size of the effects observed in these other studies leaves open the possibility that familiarity may have influenced choices in the other experiments.

One way of stating the present results is to say that generalization from a stimulus to others is based not only on physical similarity but also on their spatial-temporal similarity. Brown and Carr (1958) placed the objects to be used for the next problem 6 in. behind those being used on the current problem and the incidental objects shifted position when the correct and incorrect stimuli shifted positions. New problems were solved faster when the correct object had been paired with the correct stimulus on the preceding problem than when the correct object had been paired with an incorrect stimulus on the preceding problem. The idea that outcomes generalize to other stimuli in relation to their spatial-temporal similarity would predict such a result, and one need not infer that the monkey has some special insight into between-problem relations.

We still have little clarity, however, concerning the mechanism by which outcomes of a trial become associated with unchosen objects. One possibility is that outcomes and unchosen stimuli become directly associated, with the strength of this association depending little on the stimulus properties (and future reinforcement history) of the chosen object. An alternative possibility is that the situation is analogous to a sensory preconditioning experiment; that is, the two objects are paired prior to the choice response and then an outcome is associated with one of the stimuli (the chosen object). The unchosen stimulus may acquire strength by virtue of its pairing with the chosen stimulus. In this event, the strength of the unchosen stimulus should be closely tied to the future reward history of the chosen object. These and other explanations of the present results await further, more refined experimental analysis.

\section{REFERENCES}

BEHAR, I. Evaluation of the significance of the positive and negative cue in discrimination learning. Journal of Comparative and Physiological Psychology, 1962, 55, 502-504.

Brown, W. L., \& CARR, R. M. The learning of incidental cues by monkeys. Journal of Comparative and Physiological Psychology, 1958, 51, 459-460.

FitzGerald, R. D., \& Davis, R. T. The role of preference and 
reward in the selection of discriminanda by naive and sophisticated rhesus monkeys. Journal of Genetic Psychology, 1960, 97, 227-235.

Harlow, H. F. The formation of learning sets. Psychological Review, 1949, 56, 51-65.

LEARY, R. W. The rewarded, the unrewarded, the chosen, and the unchosen. Psychological Reports, 1956, 2, 91-97.
Medin, D. L. A theory of context in discrimination learning. In G. H. Bower (Ed.), The psychology of learning and motivation (Vol. 9). New York: Academic Press, 1975. Pp. 263-314.

(Received for publication September 15, 1976.) 